\title{
Circular cone convexity and some inequalities associated with circular cones
}

\author{
Jinchuan Zhou', Jein-Shan Chen ${ }^{2 *}$ and Hao-Feng Hung ${ }^{2}$
}

${ }^{\text {*Correspondence: }}$

jschen@math.ntnu.edu.tw

2Department of Mathematics,

National Taiwan Normal University,

Taipei, 11677, Taiwan

Full list of author information is

available at the end of the article

\begin{abstract}
The study of this paper consists of two aspects. One is characterizing the so-called circular cone convexity of $f$ by exploiting the second-order differentiability of $f \mathcal{L}_{\theta}$; the other is introducing the concepts of determinant and trace associated with circular cone and establishing their basic inequalities. These results show the essential role played by the angle $\theta$, which gives us a new insight when looking into properties about circular cone.
\end{abstract}

MSC: 26A27; 26B05; 26B35; 49J52; 90C33; 65K05

Keywords: circular cone; convexity; determinant; trace

\section{Introduction}

Recently, much attention has been paid to the nonsymmetric cone optimization problems, see [1-4] and the references therein. Unlike symmetric cones [5], there is no unified structure for nonsymmetric cones. Hence, how to tackle nonsymmetric cone optimization is still an issue. For symmetric cone optimization, the algebraic structure associated with symmetric cones, including second-order cone and positive semi-definite matrix cones, allows us to study them via exploiting the unified Euclidean Jordan algebra [5]. In general, the way to deal with nonsymmetric cone optimization depends on the feature of the associated nonsymmetric cone. In this paper, we focus on a special nonsymmetric cone, circular cone $\mathcal{L}_{\theta}$. The circular cone [6-9] is a pointed closed convex cone having hyperspherical sections orthogonal to its axis of revolution about which the cone is invariant to rotation. Let its half-aperture angle be $\theta$ with $\theta \in\left(0,90^{\circ}\right)$. Then, it is mathematically expressed as

$$
\begin{aligned}
\mathcal{L}_{\theta} & :=\left\{x=\left(x_{1}, x_{2}\right)^{T} \in \mathbb{R} \times \mathbb{R}^{n-1} \mid x_{1} \geq\|x\| \cos \theta\right\} \\
& =\left\{x=\left(x_{1}, x_{2}\right)^{T} \in \mathbb{R} \times \mathbb{R}^{n-1} \mid x_{1} \geq\left\|x_{2}\right\| \cot \theta\right\} .
\end{aligned}
$$

Real applications of a circular cone lie in some engineering problems, for example, in the formulation for optimal grasping manipulation for multi-fingered robots, the grasping force of $i$ th finger is subject to a circular cone constraint, see $[10,11]$ and references for more details.

Although $\mathcal{L}_{\theta}$ is a nonsymmetric cone, we can, due to its special structure, establish the explicit form of orthogonal decomposition (or spectral decomposition) [7] as

$$
x=\lambda_{1}(x) \cdot u_{x}^{(1)}+\lambda_{2}(x) \cdot u_{x}^{(2)}
$$

@2013 Zhou et al.; licensee Springer. This is an Open Access article distributed under the terms of the Creative Commons Attribution License (http://creativecommons.org/licenses/by/2.0), which permits unrestricted use, distribution, and reproduction in any medium, provided the original work is properly cited. 
where

$$
\left\{\begin{array}{l}
\lambda_{1}(x)=x_{1}-\left\|x_{2}\right\| \cot \theta \\
\lambda_{2}(x)=x_{1}+\left\|x_{2}\right\| \tan \theta
\end{array}\right.
$$

and

$$
\left\{\begin{array}{l}
u_{x}^{(1)}=\frac{1}{1+\cot ^{2} \theta}\left[\begin{array}{cc}
1 & 0 \\
0 \cot \theta I_{n-1}
\end{array}\right]\left[\begin{array}{c}
1 \\
-\bar{x}_{2}
\end{array}\right]=\left[\begin{array}{c}
\sin ^{2} \theta \\
-(\sin \theta \cos \theta) \bar{x}_{2}
\end{array}\right], \\
u_{x}^{(2)}=\frac{1}{1+\tan ^{2} \theta}\left[\begin{array}{cc}
1 & 0 \\
0 & \tan \theta I_{n-1}
\end{array}\right]\left[\begin{array}{c}
1 \\
\bar{x}_{2}
\end{array}\right]=\left[\begin{array}{c}
\cos ^{2} \theta \\
(\sin \theta \cos \theta) \bar{x}_{2}
\end{array}\right]
\end{array}\right.
$$

with $\bar{x}_{2}=x_{2} /\left\|x_{2}\right\|$ if $x_{2} \neq 0$, and $\bar{x}_{2}$ being any vector $w$ in $\mathbb{R}^{n-1}$ satisfying $\|w\|=1$ if $x_{2}=0$. Clearly, $x \in \mathcal{L}_{\theta}$ if and only if $\lambda_{1}(x) \geq 0$.

The formula (1) allows us to define the following vector-valued function:

$$
f^{\mathcal{L}_{\theta}}(x):=f\left(\lambda_{1}(x)\right) u_{x}^{(1)}+f\left(\lambda_{2}(x)\right) u_{x}^{(2)}
$$

where $f$ is a real-valued function from $J$ to $\mathbb{R}$ with $J$ being a subset in $\mathbb{R}$. Let $S$ be the set of all $x \in \mathbb{R}^{n}$ whose spectral values $\lambda_{i}(x)$ for $i=1,2$ belong to $J$, i.e., $S:=\left\{x \in \mathbb{R}^{n} \mid \lambda_{i}(x) \in J, i=\right.$ $1,2\}$. According to [12], we know that $S$ is open if and only if $J$ is open. In addition, as $J$ is an interval, then $S$ is convex because

$$
\begin{aligned}
\min \left\{\lambda_{1}(x), \lambda_{1}(y)\right\} & \leq \lambda_{1}(\beta x+(1-\beta) y) \leq \lambda_{2}(\beta x+(1-\beta) y) \\
& \leq \max \left\{\lambda_{2}(x), \lambda_{2}(y)\right\}, \quad \forall \beta \in[0,1] .
\end{aligned}
$$

Throughout this paper, we always assume that $J$ is an interval in $\mathbb{R}$. Clearly, as $\theta=45^{\circ}$, $\mathcal{L}_{45^{\circ}}$ reduces to the second-order cone and the above expressions (1) and (2) correspond to the spectral decomposition and the SOC-function associated with the second-order cone, respectively (see $[13,14]$ for more information regarding $f^{\text {soc }}$ ).

It is well known that in dealing with symmetric cone optimization problems, such as second-order cone optimization problems and positive semi-definite optimization problems, this type of vector-valued functions plays an essential role. Inspired by this, we study the properties of $f^{\mathcal{L}_{\theta}}$, which is crucial for circular cone optimization problems. In our previous works, we have studied the smooth and nonsmooth analysis of $f^{\mathcal{L}_{\theta}}[8,10]$; and the circular cone monotonicity and second-order differentiability of $f^{\mathcal{L}_{\theta}}$ [9]. From the aforementioned research, there is an interesting observation: some properties commonly shared by $f^{\text {soc }}$ and $f^{\mathcal{L}_{\theta}}$ are independent of the angle $\theta$; for example, $f^{\mathcal{L}_{\theta}}$ is directionally differentiable, Fréchet differentiable, semi-smooth if and only if $f$ is directionally differentiable, Fréchet differentiable, semi-smooth; while some properties are dependent on the angle $\theta$; for example, $f^{\mathcal{L}_{\theta}}$ with $f(t)=-1 / t$ for $t>0$ is circular cone monotone as $\theta \in\left[45^{\circ}, 90^{\circ}\right)$, but not circular cone monotone as $\theta \in\left(0,45^{\circ}\right)$.

In this paper, we further study the circular cone convexity of $f$. More precisely, a realvalued function $f: J \rightarrow \mathbb{R}$ is said to be $\mathcal{L}_{\theta}$-convex of order $n$ on $S$ if for any $x, y \in S$,

$$
f^{\mathcal{L}_{\theta}}(\beta x+(1-\beta) y) \preceq \mathcal{L}_{\theta} \beta f^{\mathcal{L}_{\theta}}(x)+(1-\beta) f^{\mathcal{L}_{\theta}}(y), \quad \forall \beta \in[0,1] .
$$


The characterization of $\mathcal{L}_{\theta}$-convexity is based on the observation that $f$ is $\mathcal{L}_{\theta}$-convex if and only if $\left(f^{\mathcal{L}_{\theta}}\right)^{\prime \prime}(x)(h, h) \in \mathcal{L}_{\theta}$ for all $h \in \mathbb{R}^{n}$. Our result shows that the circular cone convexity requires that the angle $\theta$ belongs in $\left[45^{\circ}, 90^{\circ}\right)$. In particular, we show that $f$ is $\mathcal{L}_{\theta}$-convex of order 2 if and only if $\theta \in\left[45^{\circ}, 90^{\circ}\right)$ and $f$ is convex.

On the other hand, using the spectral decomposition (1), we define the determinant and trace of $x$ in the framework of circular cone as

$$
\operatorname{det}(x):=\lambda_{1}(x) \lambda_{2}(x) \text { and } \operatorname{tr}(x):=\lambda_{1}(x)+\lambda_{2}(x),
$$

respectively. In the symmetric cone setting, the concepts of determinant and trace are the key ingredients of barrier and penalty functions which are used in barrier and penalty methods (including interior point methods) for symmetric cone optimization, see [1517]. Here we further study some basic inequalities of $\operatorname{det}(x)$ and $\operatorname{tr}(x)$ in the framework of circular cone. As seen in Section 3, the obtained inequalities are classified into three categories: (i) the first class is independent of the angle (i.e., still holds in the framework of circular cone); (ii) the second class is dependent on the angle, for example, for $x, y \in \mathcal{L}_{\theta}$, the inequality

$$
\operatorname{det}(e+x+y) \leq \operatorname{det}(e+x) \operatorname{det}(e+y)
$$

where $e=(1,0, \ldots, 0) \in \mathbb{R}^{n}$, fails as $\theta \in\left(0,45^{\circ}\right)$ but holds as $\theta \in\left[45^{\circ}, 90^{\circ}\right)$; (iii) the third class always fails no matter what value of $\theta$ is chosen. These results give us a new insight into a circular cone and make us focus more on the role played by the angle $\theta$.

The notation used in this paper is standard. For example, denote by $\mathbb{R}^{n}$ the $n$ dimensional Euclidean space and by $\mathbb{R}_{+}$the set of all nonnegative real scalars, i.e., $\mathbb{R}_{+}=\{t \in \mathbb{R} \mid t \geq 0\}$. For $x, y \in \mathbb{R}^{n}$, the inner product is denoted by $x^{T} y$. Let $\mathbb{S}^{n}$ mean the spaces of all real symmetric matrices in $\mathbb{R}^{n \times n}$, and let $\mathbb{S}_{+}^{n}$ denote the cone of positive semi-definite matrices. We write $x \succeq_{\mathcal{L}_{\theta}} y$ to stand for $x-y \in \mathcal{L}_{\theta}$. Finally, we define $\frac{0}{0}:=0$ for convenience.

\section{Circular cone convexity}

The main purpose of this section is to provide characterizations of $\mathcal{L}_{\theta}$-convex functions. First, we need the following technical lemma.

Lemma 2.1 Given $\alpha_{i} \in \mathbb{R}$ for $i=1, \ldots, 6$ and $\beta_{i} \in \mathbb{R}$ for $i=1,2,3$, we define

$$
\begin{aligned}
& \mathcal{F}\left(\beta_{1}, \beta_{2}, \beta_{3}\right):=\alpha_{1} \beta_{1}^{4}+\alpha_{2} \beta_{3}^{4}+\alpha_{3} \beta_{1}^{2} \beta_{3}^{2}+\alpha_{4} \beta_{2}^{2} \beta_{3}^{2}+\alpha_{5} \beta_{1}^{2} \beta_{2}^{2}+\alpha_{6} \beta_{1} \beta_{2} \beta_{3}^{2} . \\
& \text { If } \mathcal{F}\left(\beta_{1}, \beta_{2}, \beta_{3}\right) \geq 0 \text { for all }\left(\beta_{1}, \beta_{2}, \beta_{3}\right) \in \mathbb{R}^{3} \text {, then } \\
& \quad \alpha_{1} \geq 0, \quad \alpha_{2} \geq 0, \quad \alpha_{4} \geq 0, \quad \alpha_{5} \geq 0, \quad \alpha_{3} \geq-2 \sqrt{\alpha_{1} \alpha_{2}} .
\end{aligned}
$$

Furthermore, if

$$
\alpha_{6}^{2} \leq \begin{cases}4 \alpha_{2} \alpha_{5} & \text { for } \alpha_{3} \geq 0 \\ 4\left[\alpha_{2}-\left(\alpha_{3}^{2} / 4 \alpha_{1}\right)\right] \alpha_{5} & \text { for } \alpha_{3} \in\left[-2 \sqrt{\alpha_{1} \alpha_{2}}, 0\right)\end{cases}
$$

then $\mathcal{F}\left(\beta_{1}, \beta_{2}, \beta_{3}\right) \geq 0$ for all $\left(\beta_{1}, \beta_{2}, \beta_{3}\right) \in \mathbb{R}^{3}$. 
Proof If $\beta_{1}=0$, then $\mathcal{F}\left(\beta_{1}, \beta_{2}, \beta_{3}\right)=\beta_{3}^{2}\left[\alpha_{2} \beta_{3}^{2}+\alpha_{4} \beta_{2}^{2}\right]$. From $\mathcal{F}\left(\beta_{1}, \beta_{2}, \beta_{3}\right) \geq 0$, we have $\alpha_{2} \beta_{3}^{2}+\alpha_{4} \beta_{2}^{2} \geq 0$. Thus, $\alpha_{2} \geq 0$ by letting $\beta_{2} \rightarrow 0$ and $\alpha_{4} \geq 0$ by letting $\beta_{3} \rightarrow 0$.

If $\beta_{3}=0$, then $\mathcal{F}\left(\beta_{1}, \beta_{2}, \beta_{3}\right)=\beta_{1}^{2}\left[\alpha_{1} \beta_{1}^{2}+\alpha_{5} \beta_{2}^{2}\right]$. From $\mathcal{F}\left(\beta_{1}, \beta_{2}, \beta_{3}\right) \geq 0$, we obtain $\alpha_{1} \geq 0$ and $\alpha_{5} \geq 0$.

If $\beta_{2}=0$, then

$$
\mathcal{F}\left(\beta_{1}, \beta_{2}, \beta_{3}\right)=\alpha_{1} \beta_{1}^{4}+\alpha_{2} \beta_{3}^{4}+\alpha_{3} \beta_{1}^{2} \beta_{3}^{2}=\beta_{1}^{2} \beta_{3}^{2}\left[\alpha_{1}\left(\frac{\beta_{1}}{\beta_{3}}\right)^{2}+\alpha_{3}+\alpha_{2}\left(\frac{\beta_{3}}{\beta_{1}}\right)^{2}\right]
$$

whenever $\beta_{1} \neq 0$ and $\beta_{3} \neq 0$. Let $t=\beta_{1} / \beta_{3}$. From $\mathcal{F}\left(\beta_{1}, \beta_{2}, \beta_{3}\right) \geq 0$, equation (5) implies

$$
\alpha_{3} \geq-\alpha_{1} t^{2}-\alpha_{2}\left(1 / t^{2}\right), \quad \forall t \neq 0
$$

i.e.,

$$
\alpha_{3} \geq \max _{t \neq 0}\left[-\alpha_{1} t^{2}-\alpha_{2}\left(1 / t^{2}\right)\right]=-\min _{t \neq 0}\left[\alpha_{1} t^{2}+\alpha_{2}\left(1 / t^{2}\right)\right]=-2 \sqrt{\alpha_{1} \alpha_{2}} .
$$

Furthermore, if $\alpha_{3} \geq 0$, then

$$
\mathcal{F}\left(\beta_{1}, \beta_{2}, \beta_{3}\right) \geq \alpha_{2} \beta_{3}^{4}+\alpha_{5} \beta_{1}^{2} \beta_{2}^{2}+\alpha_{6} \beta_{1} \beta_{2} \beta_{3}^{2}=\left[\begin{array}{ll}
\beta_{3}^{2} & \beta_{1} \beta_{2}
\end{array}\right]\left[\begin{array}{cc}
\alpha_{2} & \alpha_{6} / 2 \\
\alpha_{6} / 2 & \alpha_{5}
\end{array}\right]\left[\begin{array}{c}
\beta_{3}^{2} \\
\beta_{1} \beta_{2}
\end{array}\right] \geq 0,
$$

where the last step is due to

$$
\left[\begin{array}{cc}
\alpha_{2} & \alpha_{6} / 2 \\
\alpha_{6} / 2 & \alpha_{5}
\end{array}\right] \succeq_{\mathbb{S}_{+}^{2}} O
$$

which is ensured by condition (4). Similarly, if $\alpha_{3} \in\left[-2 \sqrt{\alpha_{1} \alpha_{2}}, 0\right.$ ) (implying $\alpha_{1} \neq 0$ in this case), then

$$
\begin{aligned}
\mathcal{F} & \left(\beta_{1}, \beta_{2}, \beta_{3}\right) \\
& =\left(\sqrt{\alpha_{1}} \beta_{1}^{2}+\frac{\alpha_{3}}{2 \sqrt{\alpha_{1}}} \beta_{3}^{2}\right)^{2}+\left(\alpha_{2}-\frac{\alpha_{3}^{2}}{4 \alpha_{1}}\right) \beta_{3}^{4}+\alpha_{4} \beta_{2}^{2} \beta_{3}^{2}+\alpha_{5} \beta_{1}^{2} \beta_{2}^{2}+\alpha_{6} \beta_{1} \beta_{2} \beta_{3}^{2} \\
& \geq\left(\alpha_{2}-\frac{\alpha_{3}^{2}}{4 \alpha_{1}}\right) \beta_{3}^{4}+\alpha_{5} \beta_{1}^{2} \beta_{2}^{2}+\alpha_{6} \beta_{1} \beta_{2} \beta_{3}^{2} \\
& =\left[\begin{array}{ll}
\beta_{3}^{2} & \beta_{1} \beta_{2}
\end{array}\right]\left[\begin{array}{cc}
\alpha_{2}-\left(\alpha_{3}^{2} / 4 \alpha_{1}\right) & \alpha_{6} / 2 \\
\alpha_{6} / 2 & \alpha_{5}
\end{array}\right]\left[\begin{array}{c}
\beta_{3}^{2} \\
\beta_{1} \beta_{2}
\end{array}\right] \geq 0,
\end{aligned}
$$

where the last step is due to

$$
\left[\begin{array}{cc}
\alpha_{2}-\left(\alpha_{3}^{2} / 4 \alpha_{1}\right) & \alpha_{6} / 2 \\
\alpha_{6} / 2 & \alpha_{5}
\end{array}\right] \succeq_{\mathbb{S}_{+}^{2}} O,
$$

which is ensured by condition (4) and the fact $\alpha_{2}-\left(\alpha_{3}^{2} / 4 \alpha_{1}\right) \geq 0$ since $-2 \sqrt{\alpha_{1} \alpha_{2}} \leq \alpha_{3}<0$. This completes the proof.

Lemma 2.2 [9, Theorem 3.1] Letf $: J \rightarrow \mathbb{R}$ and $f^{\mathcal{L}_{\theta}}$ be defined as in (2). Then $f^{\mathcal{L}_{\theta}}$ is secondorder differentiable at $x \in S$ if and only iff is second-order differentiable at $\lambda_{i}(x) \in J$ for 
$i=1,2$. Moreover, for $u, v \in \mathbb{R}^{n}$, if $x_{2}=0$, then

$$
\begin{aligned}
& \left(f^{\mathcal{L}_{\theta}}\right)^{\prime \prime}(x)(u, v) \\
& = \begin{cases}f^{\prime \prime}\left(x_{1}\right)\left[\begin{array}{c}
u^{T} v \\
u_{1} v_{2}+v_{1} u_{2}
\end{array}\right], & \text { either } u_{2}=0 \text { or } v_{2}=0, \\
{\left[\begin{array}{ll}
f^{\prime \prime}\left(x_{1}\right)\left(v_{1} u_{2}+u_{1} v_{2}\right)+\frac{1}{2} f^{\prime \prime}\left(x_{1}\right)(\tan \theta-\cot \theta)\left(\left\|u_{2}\right\| v_{2}+\bar{u}_{2}^{T} \bar{v}_{2}\left\|v_{2}\right\| u_{2}\right)
\end{array}\right],} & \text { otherwise. }\end{cases}
\end{aligned}
$$

If $x_{2} \neq 0$, then

$$
\left(f^{\mathcal{L}_{\theta}}\right)^{\prime \prime}(x)(u, v)=\left[\begin{array}{l}
I_{1} \\
I_{2}
\end{array}\right],
$$

where

$$
\begin{aligned}
I_{1}:= & v_{1} u_{1} \tilde{\xi}+\tilde{\varrho}\left(u_{1} \bar{x}_{2}^{T} v_{2}+v_{1} \bar{x}_{2}^{T} u_{2}\right)+\tilde{a} v_{2}^{T} u_{2}+(\tilde{\eta}-\tilde{a}) \bar{x}_{2}^{T} v_{2} \bar{x}_{2}^{T} u_{2}, \\
I_{2}:= & {\left[(\tilde{\eta}-\tilde{a}) u_{1} \bar{x}_{2}^{T} v_{2}+(\varpi-3 \tilde{d}) \bar{x}_{2}^{T} v_{2} \bar{x}_{2}^{T} u_{2}+\tilde{\varrho} v_{1} u_{1}+(\tilde{\eta}-\tilde{a}) v_{1} \bar{x}_{2}^{T} u_{2}\right] \bar{x}_{2} } \\
& +\tilde{d}\left[\bar{x}_{2}^{T} u_{2} v_{2}+v_{2}^{T} u_{2} \bar{x}_{2}+\bar{x}_{2}^{T} v_{2} u_{2}\right]+\tilde{a}\left(u_{1} v_{2}+v_{1} u_{2}\right)
\end{aligned}
$$

with

$$
\begin{aligned}
& \tilde{a}=\frac{f^{\prime}\left(\lambda_{2}(x)\right)-f^{\prime}\left(\lambda_{1}(x)\right)}{\lambda_{2}(x)-\lambda_{1}(x)}, \\
& \tilde{\xi}=\frac{f^{\prime \prime}\left(\lambda_{1}(x)\right)}{1+\cot ^{2} \theta}+\frac{f^{\prime \prime}\left(\lambda_{2}(x)\right)}{1+\tan ^{2} \theta}, \\
& \tilde{\varrho}=-\frac{\cot \theta}{1+\cot ^{2} \theta} f^{\prime \prime}\left(\lambda_{1}(x)\right)+\frac{\tan \theta}{1+\tan ^{2} \theta} f^{\prime \prime}\left(\lambda_{2}(x)\right), \\
& \tilde{\eta}=\frac{\cot ^{2} \theta}{1+\cot ^{2} \theta} f^{\prime \prime}\left(\lambda_{1}(x)\right)+\frac{\tan ^{2} \theta}{1+\tan ^{2} \theta} f^{\prime \prime}\left(\lambda_{2}(x)\right), \\
& \tilde{d}=\frac{1}{\left\|x_{2}\right\|}\left[\frac{\cot ^{2} \theta}{1+\cot ^{2} \theta} f^{\prime}\left(\lambda_{1}(x)\right)+\frac{\tan ^{2} \theta}{1+\tan ^{2} \theta} f^{\prime}\left(\lambda_{2}(x)\right)-\frac{f\left(\lambda_{2}(x)\right)-f\left(\lambda_{1}(x)\right)}{\lambda_{2}(x)-\lambda_{1}(x)}\right], \\
& \varpi=-\frac{\cot ^{3} \theta}{1+\cot ^{2} \theta} f^{\prime \prime}\left(\lambda_{1}(x)\right)+\frac{\tan ^{3} \theta}{1+\tan ^{2} \theta} f^{\prime \prime}\left(\lambda_{2}(x)\right) .
\end{aligned}
$$

The characterization of $\mathcal{L}_{\theta}$-convexity is established below, which can be regarded as the extension of some results given in $[12,18-20]$ from the second-order cone setting to the circular cone setting.

Theorem 2.1 Suppose that $f: J \rightarrow \mathbb{R}$ is second-order continuously differentiable. If $f$ is $\mathcal{L}_{\theta}$-convex of order $n$ on $S$, then $\tan \theta \geq 1, f$ is convex on $J$, and for all $\tau_{1}, \tau_{2} \in J$ with $\tau_{1} \leq \tau_{2}$,

$$
f^{\prime \prime}\left(\tau_{2}\right) \delta\left(\tau_{2}, \tau_{1}\right) \geq \frac{2}{\left(\tau_{2}-\tau_{1}\right)^{2}} \delta\left(\tau_{1}, \tau_{2}\right)^{2}
$$

and

$$
\begin{aligned}
& {\left[\tan ^{2} \theta \delta\left(\tau_{1}, \tau_{2}\right)+\left(\tan ^{2} \theta-1\right) \delta\left(\tau_{2}, \tau_{1}\right)\right] f^{\prime \prime}\left(\tau_{1}\right)-\frac{2}{\left(\tau_{2}-\tau_{1}\right)^{2}} \delta\left(\tau_{2}, \tau_{1}\right)^{2}} \\
& \quad \geq-f^{\prime \prime}\left(\tau_{1}\right) \sqrt{\left(\tan ^{2} \theta-1\right) \delta\left(\tau_{2}, \tau_{1}\right)\left[2 \tan ^{2} \theta \delta\left(\tau_{1}, \tau_{2}\right)+\left(\tan ^{2} \theta-1\right) \delta\left(\tau_{2}, \tau_{1}\right)\right]} .
\end{aligned}
$$


Furthermore, if

$$
\left[\tan ^{2} \theta \delta\left(\tau_{1}, \tau_{2}\right)+\left(\tan ^{2} \theta-1\right) \delta\left(\tau_{2}, \tau_{1}\right)\right] f^{\prime \prime}\left(\tau_{1}\right) \geq \frac{2}{\left(\tau_{2}-\tau_{1}\right)^{2}} \delta\left(\tau_{2}, \tau_{1}\right)^{2}
$$

and

$$
8 \delta\left(\tau_{2}, \tau_{1}\right) \delta\left(\tau_{1}, \tau_{2}\right)^{2} \leq\left[2 \tan ^{2} \theta \delta\left(\tau_{1}, \tau_{2}\right)+\left(\tan ^{2} \theta-1\right) \delta\left(\tau_{2}, \tau_{1}\right)\right] f^{\prime \prime}\left(\tau_{1}\right) f^{\prime \prime}\left(\tau_{2}\right)\left(\tau_{2}-\tau_{1}\right)^{4},
$$

or if

$$
\left[\tan ^{2} \theta \delta\left(\tau_{1}, \tau_{2}\right)+\left(\tan ^{2} \theta-1\right) \delta\left(\tau_{2}, \tau_{1}\right)\right] f^{\prime \prime}\left(\tau_{1}\right)<\frac{2}{\left(\tau_{2}-\tau_{1}\right)^{2}} \delta\left(\tau_{2}, \tau_{1}\right)^{2}
$$

and

$$
\begin{aligned}
& 8 \delta\left(\tau_{1}, \tau_{2}\right)^{2} \delta\left(\tau_{2}, \tau_{1}\right)^{2}\left(\tan ^{2} \theta-1\right) f^{\prime \prime}\left(\tau_{1}\right) \\
& \leq\left\{\left[\left(\tan ^{2} \theta-1\right) f^{\prime \prime}\left(\tau_{1}\right)^{2}\left[2 \tan ^{2} \theta \delta\left(\tau_{1}, \tau_{2}\right)+\left(\tan ^{2} \theta-1\right) \delta\left(\tau_{2}, \tau_{1}\right)\right] \delta\left(\tau_{2}, \tau_{1}\right)\right]\right. \\
& \left.\quad-\left[\left(\tan ^{2} \theta \delta\left(\tau_{1}, \tau_{2}\right)+\left(\tan ^{2} \theta-1\right) \delta\left(\tau_{2}, \tau_{1}\right)\right) f^{\prime \prime}\left(\tau_{1}\right)-\frac{2}{\left(\tau_{2}-\tau_{1}\right)^{2}} \delta\left(\tau_{2}, \tau_{1}\right)^{2}\right]^{2}\right\} \\
& \quad \times f^{\prime \prime}\left(\tau_{2}\right)\left(\tau_{2}-\tau_{1}\right)^{4},
\end{aligned}
$$

then $f$ is $\mathcal{L}_{\theta}$-convex. Here $\delta\left(\tau, \tau^{\prime}\right):=f(\tau)-f\left(\tau^{\prime}\right)-f^{\prime}\left(\tau^{\prime}\right)\left(\tau-\tau^{\prime}\right)$ for $\tau, \tau^{\prime} \in J$.

Proof According to [9, Theorem 3.2], $f$ is $\mathcal{L}_{\theta}$-convex if and only if $\left(f^{\mathcal{L}_{\theta}}\right)^{\prime \prime}(x)(h, h) \in \mathcal{L}_{\theta}$ for all $x \in S$ and $h \in \mathbb{R}^{n}$. We proceed the proof by considering the following three cases.

Case 1. For $x_{2}=0$ and $h_{2}=0$, it follows from Lemma 2.2 that

$$
\left(f^{\mathcal{L}_{\theta}}\right)^{\prime \prime}(x)(h, h)=f^{\prime \prime}\left(x_{1}\right)\left[\begin{array}{c}
h_{1}^{2} \\
0
\end{array}\right]
$$

Hence, $\left(f^{\mathcal{L}_{\theta}}\right)^{\prime \prime}(x)(h, h) \in \mathcal{L}_{\theta}$ if and only if $f^{\prime \prime}\left(x_{1}\right) \geq 0$.

Case 2. For $x_{2}=0$ and $h_{2} \neq 0$, it follows from Lemma 2.2 that

$$
\left(f^{\mathcal{L}_{\theta}}\right)^{\prime \prime}(x)(h, h)=\left[\begin{array}{c}
f^{\prime \prime}\left(x_{1}\right)\|h\|^{2} \\
2 f^{\prime \prime}\left(x_{1}\right) h_{1} h_{2}+f^{\prime \prime}\left(x_{1}\right)(\tan \theta-\cot \theta)\left\|h_{2}\right\| h_{2}
\end{array}\right] .
$$

Hence, $\left(f^{\mathcal{L}_{\theta}}\right)^{\prime \prime}(x)(h, h) \in \mathcal{L}_{\theta}$ if and only if $f^{\prime \prime}\left(x_{1}\right) \geq 0$ and

$$
\tan \theta\|h\|^{2} \geq\left|2 h_{1}+(\tan \theta-\cot \theta)\left\|h_{2}\right\|\right| \mid\left\|h_{2}\right\|,
$$

i.e.,

$$
-\tan \theta\left(h_{1}^{2}+\left\|h_{2}\right\|^{2}\right) \leq\left[2 h_{1}+(\tan \theta-\cot \theta)\left\|h_{2}\right\|\right]\left\|h_{2}\right\| \leq \tan \theta\left(h_{1}^{2}+\left\|h_{2}\right\|^{2}\right) .
$$


Dividing by $\left\|h_{2}\right\|^{2}$ and letting $t=h_{1} /\left\|h_{2}\right\|$ yields

$$
\begin{aligned}
- & \tan \theta\left(t^{2}+1\right) \leq 2 t+\tan \theta-\cot \theta \leq \tan \theta\left(t^{2}+1\right) \\
& \Longleftrightarrow \max _{t \in \mathbb{R}}-\tan \theta\left(t^{2}+1\right)-2 t \leq \tan \theta-\cot \theta \leq \min _{t \in \mathbb{R}} \tan \theta\left(t^{2}+1\right)-2 t \\
& \Longleftrightarrow \cot \theta-\tan \theta \leq \tan \theta-\cot \theta \leq \tan \theta-\cot \theta \\
& \Longleftrightarrow \tan \theta \geq 1 .
\end{aligned}
$$

Case 3 . For $x_{2} \neq 0$, due to the simplification of notation, let us denote

$$
\mu_{1}:=h_{1}-\cot \theta \bar{x}_{2}^{T} h_{2}, \quad \mu_{2}:=h_{1}+\tan \theta \bar{x}_{2}^{T} h_{2}, \quad \mu_{3}:=\sqrt{\left\|h_{2}\right\|^{2}-\left(\bar{x}_{2}^{T} h_{2}\right)^{2}} .
$$

Then

$$
\bar{x}_{2}^{T} h_{2}=\frac{\mu_{2}-\mu_{1}}{\tan \theta+\cot \theta} \text { and } \quad h_{1}=\frac{\tan \theta \mu_{1}+\cot \theta \mu_{2}}{\tan \theta+\cot \theta} .
$$

Note that $\mu_{1}, \mu_{2}$, and $\mu_{3}$ can take any value in $\mathbb{R} \times \mathbb{R} \times \mathbb{R}_{+}$by taking a suitable value of $h$ (because the vector $h$ has $n$ variables). It follows from Lemma 2.2 that

$$
\begin{aligned}
& \left(f^{\mathcal{L}_{\theta}}\right)^{\prime \prime}(x)(h, h) \\
& =\left[\begin{array}{c}
\tilde{\xi} h_{1}^{2}+2 \tilde{\varrho} \bar{x}_{2}^{T} h_{2} h_{1}+\tilde{a}\left\|h_{2}\right\|^{2}+(\tilde{\eta}-\tilde{a})\left(\bar{x}_{2}^{T} h_{2}\right)^{2} \\
\left.\left[(\varpi-3 \tilde{d})\left(\bar{x}_{2}^{T} h_{2}\right)^{2}+2(\tilde{\eta}-\tilde{a}) \bar{x}_{2}^{T} h_{2} h_{1}\right] \bar{x}_{2}+\left[\tilde{\varrho} h_{1}^{2}+\tilde{d}\left\|h_{2}\right\|^{2}\right] \bar{x}_{2}+2\left[\tilde{a} h_{1}+\tilde{d} \bar{x}_{2}^{T} h_{2}\right] h_{2}\right] \\
=: \\
\Theta_{2} \bar{x}_{2}+\Theta_{3} h_{2}
\end{array}\right],
\end{aligned}
$$

where

$$
\begin{aligned}
& \Theta_{1}=\tilde{\xi} h_{1}^{2}+2 \tilde{\varrho} \bar{x}_{2}^{T} h_{2} h_{1}+\tilde{a}\left\|h_{2}\right\|^{2}+(\tilde{\eta}-\tilde{a})\left(\bar{x}_{2}^{T} h_{2}\right)^{2}, \\
& \Theta_{2}=(\varpi-3 \tilde{d})\left(\bar{x}_{2}^{T} h_{2}\right)^{2}+2(\tilde{\eta}-\tilde{a}) \bar{x}_{2}^{T} h_{2} h_{1}+\tilde{\varrho} h_{1}^{2}+\tilde{d}\left\|h_{2}\right\|^{2}, \\
& \Theta_{3}=2\left[\tilde{a} h_{1}+\tilde{d} \bar{x}_{2}^{T} h_{2}\right] .
\end{aligned}
$$

Hence, $\left(f^{\mathcal{L}_{\theta}}\right)^{\prime \prime}(x)(h, h) \in \mathcal{L}_{\theta}$ is equivalent to

$$
\Theta_{1} \geq 0 \text { and } \Theta_{1}^{2} \tan ^{2} \theta \geq\left\|\Theta_{2} \bar{x}_{2}+\Theta_{3} h_{2}\right\|^{2} .
$$

Note that

$$
\begin{aligned}
\Theta_{1}= & \frac{1}{1+\cot ^{2} \theta} f^{\prime \prime}\left(\lambda_{1}(x)\right)\left[h_{1}^{2}-2\left(\bar{x}_{2}^{T} h_{2}\right) h_{1} \cot \theta+\left(\bar{x}_{2}^{T} h_{2}\right)^{2} \cot ^{2} \theta\right] \\
& +\frac{1}{1+\tan ^{2} \theta} f^{\prime \prime}\left(\lambda_{2}(x)\right)\left[h_{1}^{2}+2\left(\bar{x}_{2}^{T} h_{2}\right) h_{1} \tan \theta+\left(\bar{x}_{2}^{T} h_{2}\right)^{2} \tan ^{2} \theta\right] \\
& +\tilde{a}\left[\left\|h_{2}\right\|^{2}-\left(\bar{x}_{2}^{T} h_{2}\right)^{2}\right] \\
= & \frac{1}{1+\cot ^{2} \theta} f^{\prime \prime}\left(\lambda_{1}(x)\right) \mu_{1}^{2}+\frac{1}{1+\tan ^{2} \theta} f^{\prime \prime}\left(\lambda_{2}(x)\right) \mu_{2}^{2}+\tilde{a} \mu_{3}^{2} .
\end{aligned}
$$


We now claim that $\Theta_{1} \geq 0$ for all $h \in \mathbb{R}^{n}$ if and only if

$$
f^{\prime \prime}\left(\lambda_{1}(x)\right) \geq 0, \quad f^{\prime \prime}\left(\lambda_{2}(x)\right) \geq 0, \quad \text { and } \quad \tilde{a} \geq 0 .
$$

The sufficiency is clear. Let us show the necessity. In particular, choosing $h=\left(-\tan \theta, \bar{x}_{2}\right)$ yields $\mu_{2}=0$ and $\mu_{3}=0$. It then follows from $\Theta_{1} \geq 0$ that $f^{\prime \prime}\left(\lambda_{1}(x)\right) \geq 0$. If we choose $h=\left(\cot \theta, \bar{x}_{2}\right)$, then we have $f^{\prime \prime}\left(\lambda_{2}(x)\right) \geq 0$. Finally, choosing $h=\left(1, k z_{2}\right)$ with $k \in \mathbb{R},\left\|z_{2}\right\|=1$ and $z_{2}^{T} \bar{x}_{2}=0$ gives

$$
\Theta_{1}=\frac{f^{\prime \prime}\left(\lambda_{1}(x)\right)}{1+\cot ^{2} \theta}+\frac{f^{\prime \prime}\left(\lambda_{2}(x)\right)}{1+\tan ^{2} \theta}+\tilde{a} k^{2} \geq 0 .
$$

Dividing by $k^{2}$ both sides and taking the limits as $k \rightarrow \infty$, we obtain $\tilde{a} \geq 0$. Since $\lambda_{i}(x)$ can take an arbitrary value in $J$, it is clear that (14) is equivalent to saying that $f^{\prime \prime}(\tau) \geq 0$ for all $\tau \in J$, i.e., $f$ is convex on $J$. Indeed, the condition $\tilde{a} \geq 0$ is ensured by the fact that $\tilde{a}=\frac{f^{\prime}\left(\lambda_{2}(x)\right)-f^{\prime}\left(\lambda_{1}(x)\right)}{\lambda_{2}(x)-\lambda_{1}(x)}=f^{\prime \prime}\left(t_{0}\right) \geq 0$ for some $t_{0} \in\left(\lambda_{1}(x), \lambda_{2}(x)\right)$.

Now we calculate the values of $\Theta_{2}$ and $\Theta_{3}$, respectively.

$$
\begin{aligned}
\Theta_{2}= & -\frac{\cot \theta}{1+\cot ^{2} \theta} f^{\prime \prime}\left(\lambda_{1}(x)\right) \mu_{1}^{2}+\frac{\tan \theta}{1+\tan ^{2} \theta} f^{\prime \prime}\left(\lambda_{2}(x)\right) \mu_{2}^{2} \\
& +\tilde{d} \mu_{3}^{2}-2\left(\tilde{d} \bar{x}_{2}^{T} h_{2}+\tilde{a} h_{1}\right)\left(\bar{x}_{2}^{T} h_{2}\right) \\
= & -\frac{\cot \theta}{1+\cot ^{2} \theta} f^{\prime \prime}\left(\lambda_{1}(x)\right) \mu_{1}^{2}+\frac{\tan \theta}{1+\tan ^{2} \theta} f^{\prime \prime}\left(\lambda_{2}(x)\right) \mu_{2}^{2}+\tilde{d} \mu_{3}^{2}-\left(\bar{x}_{2}^{T} h_{2}\right) \Theta_{3} .
\end{aligned}
$$

Meanwhile, it follows from (12) that

$$
\begin{aligned}
\Theta_{3} & =2\left[\tilde{a} \frac{\tan \theta \mu_{1}+\cot \theta \mu_{2}}{\tan \theta+\cot \theta}+\tilde{d} \frac{\mu_{2}-\mu_{1}}{\tan \theta+\cot \theta}\right] \\
& =\frac{2}{\tan \theta+\cot \theta}\left[\mu_{1}(\tilde{a} \tan \theta-\tilde{d})+\mu_{2}(\tilde{a} \cot \theta+\tilde{d})\right] .
\end{aligned}
$$

Note that

$$
\begin{aligned}
\left\|\Theta_{2} \bar{x}_{2}+\Theta_{3} h_{2}\right\|^{2} & =\Theta_{2}^{2}+2 \Theta_{2} \Theta_{3} \bar{x}_{2}^{T} h_{2}+\Theta_{3}^{2}\left\|h_{2}\right\|^{2} \\
& =\Theta_{2}^{2}+2 \Theta_{2} \Theta_{3} \bar{x}_{2}^{T} h_{2}+\Theta_{3}^{2}\left[\mu_{3}^{2}+\left(\bar{x}_{2}^{T} h_{2}\right)^{2}\right] \\
& =\left(\Theta_{2}+\Theta_{3} \bar{x}_{2}^{T} h_{2}\right)^{2}+\Theta_{3}^{2} \mu_{3}^{2} .
\end{aligned}
$$

Putting (13) and (15)-(17) together, the condition $\Theta_{1}^{2} \tan ^{2} \theta \geq\left\|\Theta_{2} \bar{x}_{2}+\Theta_{3} h_{2}\right\|^{2}$ can be rewritten equivalently as

$$
\begin{aligned}
\tan ^{2} \theta & {\left[\frac{f^{\prime \prime}\left(\lambda_{1}(x)\right)}{1+\cot ^{2} \theta} \mu_{1}^{2}+\frac{f^{\prime \prime}\left(\lambda_{2}(x)\right)}{1+\tan ^{2} \theta} \mu_{2}^{2}+\tilde{a} \mu_{3}^{2}\right]^{2} } \\
\geq & {\left[-\frac{\cot \theta}{1+\cot ^{2} \theta} f^{\prime \prime}\left(\lambda_{1}(x)\right) \mu_{1}^{2}+\frac{\tan \theta}{1+\tan ^{2} \theta} f^{\prime \prime}\left(\lambda_{2}(x)\right) \mu_{2}^{2}+\tilde{d} \mu_{3}^{2}\right]^{2} } \\
& +\frac{4}{(\tan \theta+\cot \theta)^{2}}\left[\mu_{1}(\tilde{a} \tan \theta-\tilde{d})+\mu_{2}(\tilde{a} \cot \theta+\tilde{d})\right]^{2} \mu_{3}^{2},
\end{aligned}
$$


i.e.,

$$
\begin{aligned}
& \left(\tan ^{4} \theta-1\right) f^{\prime \prime}\left(\lambda_{1}(x)\right)^{2} \mu_{1}^{4}+(\tan \theta+\cot \theta)^{2}\left(\tilde{a}^{2} \tan ^{2} \theta-\tilde{d}^{2}\right) \mu_{3}^{4} \\
& +2\left[(\tan \theta+\cot \theta)\left(\tilde{a} \tan ^{3} \theta+\tilde{d}\right) f^{\prime \prime}\left(\lambda_{1}(x)\right)-2(\tilde{a} \tan \theta-\tilde{d})^{2}\right] \mu_{1}^{2} \mu_{3}^{2} \\
& +2\left[(\tan \theta+\cot \theta)(\tilde{a} \tan \theta-\tilde{d}) f^{\prime \prime}\left(\lambda_{2}(x)\right)-2(\tilde{a} \cot \theta+\tilde{d})^{2}\right] \mu_{2}^{2} \mu_{3}^{2} \\
& +2\left(\tan ^{2} \theta+1\right) f^{\prime \prime}\left(\lambda_{1}(x)\right) f^{\prime \prime}\left(\lambda_{2}(x)\right) \mu_{1}^{2} \mu_{2}^{2} \\
& \quad-8(\tilde{a} \tan \theta-\tilde{d})(\tilde{a} \cot \theta+\tilde{d}) \mu_{1} \mu_{2} \mu_{3}^{2} \geq 0 .
\end{aligned}
$$

To apply Lemma 2.1, we need to compute each coefficient in (18). By calculation, we have

$$
\begin{aligned}
\tilde{a} \tan \theta & -\tilde{d} \\
= & \frac{f^{\prime}\left(\lambda_{2}(x)\right)-f^{\prime}\left(\lambda_{1}(x)\right)}{\lambda_{2}(x)-\lambda_{1}(x)} \tan \theta-\frac{1}{\left\|x_{2}\right\|}\left[\frac{\cot ^{2} \theta}{1+\cot ^{2} \theta} f^{\prime}\left(\lambda_{1}(x)\right)+\frac{\tan ^{2} \theta}{1+\tan ^{2} \theta} f^{\prime}\left(\lambda_{2}(x)\right)\right] \\
& +\frac{1}{\left\|x_{2}\right\|} \frac{f\left(\lambda_{2}(x)\right)-f\left(\lambda_{1}(x)\right)}{\lambda_{2}(x)-\lambda_{1}(x)} \\
= & \frac{f^{\prime}\left(\lambda_{2}(x)\right)-f^{\prime}\left(\lambda_{1}(x)\right)}{\lambda_{2}(x)-\lambda_{1}(x)} \tan \theta-\frac{1}{\left\|x_{2}\right\|}\left[\frac{\cot \theta}{\tan \theta+\cot \theta} f^{\prime}\left(\lambda_{1}(x)\right)+\frac{\tan \theta}{\tan \theta+\operatorname{ctan} \theta} f^{\prime}\left(\lambda_{2}(x)\right)\right] \\
& +\frac{1}{\left\|x_{2}\right\|} \frac{f\left(\lambda_{2}(x)\right)-f\left(\lambda_{1}(x)\right)}{\lambda_{2}(x)-\lambda_{1}(x)} \\
= & -\frac{\tan \theta+\operatorname{ctan} \theta}{\lambda_{2}(x)-\lambda_{1}(x)} f^{\prime}\left(\lambda_{1}(x)\right)+\frac{\tan \theta+\operatorname{ctan} \theta}{\left[\lambda_{2}(x)-\lambda_{1}(x)\right]^{2}}\left[f\left(\lambda_{2}(x)\right)-f\left(\lambda_{1}(x)\right)\right] \\
= & \frac{(\tan \theta+\cot \theta)\left[f\left(\lambda_{2}(x)\right)-f\left(\lambda_{1}(x)\right)-f^{\prime}\left(\lambda_{1}(x)\right)\left(\lambda_{2}(x)-\lambda_{1}(x)\right)\right]}{\left[\lambda_{2}(x)-\lambda_{1}(x)\right]^{2}} \\
= & \frac{\tan \theta+\cot \theta}{\left[\lambda_{2}(x)-\lambda_{1}(x)\right]^{2}} \delta\left(\lambda_{2}(x), \lambda_{1}(x)\right),
\end{aligned}
$$

where the third equation follows from the fact $\lambda_{2}(x)-\lambda_{1}(x)=(\tan \theta+\operatorname{ctan} \theta)\left\|x_{2}\right\|$. Similarly, we have

$$
\begin{aligned}
& \tilde{a} \tan \theta+\tilde{d} \\
& =\frac{(\tan \theta+\cot \theta)\left[f\left(\lambda_{1}(x)\right)-f\left(\lambda_{2}(x)\right)+\left(\frac{2 \tan \theta}{\tan \theta+\cot \theta} f^{\prime}\left(\lambda_{2}(x)\right)+\frac{\cot \theta-\tan \theta}{\tan \theta+\cot \theta} f^{\prime}\left(\lambda_{1}(x)\right)\right)\left(\lambda_{2}(x)-\lambda_{1}(x)\right)\right]}{\left[\lambda_{2}(x)-\lambda_{1}(x)\right]^{2}} \\
& =\frac{\tan \theta+\cot \theta}{\left[\lambda_{2}(x)-\lambda_{1}(x)\right]^{2}}\left[\frac{2 \tan ^{2} \theta}{\tan ^{2}+1} \delta\left(\lambda_{1}(x), \lambda_{2}(x)\right)+\frac{\tan ^{2} \theta-1}{\tan ^{2} \theta+1} \delta\left(\lambda_{2}(x), \lambda_{1}(x)\right)\right], \\
& \tilde{a} \cot \theta+\tilde{d}=\frac{(\tan \theta+\cot \theta)\left[f\left(\lambda_{1}(x)\right)-f\left(\lambda_{2}(x)\right)-f^{\prime}\left(\lambda_{2}(x)\right)\left(\lambda_{1}(x)-\lambda_{2}(x)\right)\right]}{\left[\lambda_{2}(x)-\lambda_{1}(x)\right]^{2}} \\
& \quad=\frac{\tan \theta+\cot \theta}{\left[\lambda_{2}(x)-\lambda_{1}(x)\right]^{2}} \delta\left(\lambda_{1}(x), \lambda_{2}(x)\right), \\
& \quad \tilde{a} \tan ^{3} \theta+\tilde{d} \\
& \quad=\frac{(\tan \theta+\cot \theta)\left[f\left(\lambda_{1}(x)\right)-f\left(\lambda_{2}(x)\right)-\left[\tan ^{2} \theta f^{\prime}\left(\lambda_{2}(x)\right)+\left(1-\tan ^{2} \theta\right) f^{\prime}\left(\lambda_{1}(x)\right)\right]\left(\lambda_{1}(x)-\lambda_{2}(x)\right)\right]}{\left[\lambda_{2}(x)-\lambda_{1}(x)\right]^{2}} \\
& \quad=\frac{\tan \theta+\cot \theta}{\left[\lambda_{2}(x)-\lambda_{1}(x)\right]^{2}}\left[\tan ^{2} \theta \delta\left(\lambda_{1}(x), \lambda_{2}(x)\right)+\left(\tan ^{2} \theta-1\right) \delta\left(\lambda_{2}(x), \lambda_{1}(x)\right)\right] .
\end{aligned}
$$


Corresponding each coefficient in (18) to (3), we know

$$
\left\{\begin{aligned}
\alpha_{1}= & (\tan 4 \theta-1) f^{\prime \prime}\left(\lambda_{1}(x)\right)^{2}, \\
\alpha_{2}= & \frac{(\tan \theta+\cot \theta)^{4}}{\left[\lambda_{2}(x)-\lambda_{1}(x)\right]^{4}} \delta\left(\lambda_{2}(x), \lambda_{1}(x)\right)\left[\frac{2 \tan ^{2} \theta}{\tan ^{2} \theta+1} \delta\left(\lambda_{1}(x), \lambda_{2}(x)\right)+\frac{\tan ^{2} \theta-1}{\tan ^{2} \theta+1} \delta\left(\lambda_{2}(x), \lambda_{1}(x)\right)\right], \\
\alpha_{3}= & 2 \frac{(\tan \theta+\cot \theta)^{2}}{\left[\lambda_{2}(x)-\lambda_{1}(x)\right]^{2}}\left\{\left[\tan ^{2} \theta \delta\left(\lambda_{1}(x), \lambda_{2}(x)\right)+\left(\tan ^{2} \theta-1\right) \delta\left(\lambda_{2}(x), \lambda_{1}(x)\right)\right] f^{\prime \prime}\left(\lambda_{1}(x)\right),\right. \\
& \left.-2 \frac{\delta\left(\lambda_{2}(x), \lambda_{1}(x)\right)^{2}}{\left[\lambda_{2}(x)-\lambda_{1}(x)\right]^{2}}\right\} \\
\alpha_{4}= & 2 \frac{(\tan \theta+\cot \theta)^{2}}{\left[\lambda_{2}(x)-\lambda_{1}(x)\right]^{2}}\left[\delta\left(\lambda_{2}(x), \lambda_{1}(x)\right) f^{\prime \prime}\left(\lambda_{2}(x)\right)-2 \frac{\delta\left(\lambda_{1}(x), \lambda_{2}(x)\right)^{2}}{\left[\lambda_{2}(x)-\lambda_{1}(x)\right]^{2}}\right], \\
\alpha_{5}= & 2\left(\tan { }^{2} \theta+1\right) f^{\prime \prime}\left(\lambda_{1}(x)\right) f^{\prime \prime}\left(\lambda_{2}(x)\right), \\
\alpha_{6}= & -8 \frac{(\tan \theta+\cot \theta)^{2}}{\left[\lambda_{2}(x)-\lambda_{1}(x)\right]^{2}} \delta\left(\lambda_{1}(x), \lambda_{2}(x)\right) \delta\left(\lambda_{2}(x), \lambda_{1}(x)\right) .
\end{aligned}\right.
$$

In view of Lemma 2.1, the condition $\alpha_{1} \geq 0$ means $\tan \theta \geq 1, \alpha_{2}, \alpha_{5} \geq 0$ is ensured by the convexity of $f$ (see (14)), $\alpha_{4} \geq 0$ corresponds to (6), and $\alpha_{3} \geq-2 \sqrt{\alpha_{1} \alpha_{2}}$ corresponds to (7). In addition, condition (4) takes the special form (9) and (10), respectively.

Theorem 2.2 Suppose that $f: J \rightarrow \mathbb{R}$ is second-order continuously differentiable. Then $f$ is $\mathcal{L}_{\theta}$-convex of order 2 on $S$ if and only if $\tan \theta \geq 1$ and $f$ is convex on $J$.

Proof The necessity is clear from Theorem 2.1. For sufficiency, note that in (11) $\mu_{3}=0$ since $\bar{x}_{2}= \pm 1$ in this case. Hence, (18) takes the form of

$$
\left(\tan ^{4} \theta-1\right) f^{\prime \prime}\left(\lambda_{1}(x)\right)^{2} \mu_{1}^{4}+2\left(\tan ^{2} \theta+1\right) f^{\prime \prime}\left(\lambda_{1}(x)\right) f^{\prime \prime}\left(\lambda_{2}(x)\right) \mu_{1}^{2} \mu_{2}^{2} \geq 0
$$

for all $\mu_{1}$ and $\mu_{2}$, which is equivalent to verifying

$$
\tan \theta \geq 1 \quad \text { and } f^{\prime \prime}\left(\lambda_{1}(x)\right) f^{\prime \prime}\left(\lambda_{2}(x)\right) \geq 0 .
$$

This is ensued by the conditions that $\tan \theta \geq 1$ and $f$ is convex on $J$. Thus, the proof is complete.

If, in particular, $\theta=45^{\circ}$, then (6) and (7) reduce to [12, (21) in Proposition 4.2]; (9) reduces to [12, (22) in Proposition 4.2]. In addition, due to (7), (8) holds automatically in this case. The above results indicate that the $\mathcal{L}_{\theta}$-convexity is dependent on the properties of $f$ and the angle $\theta$ together.

\section{Inequalities associated with circular cone}

In this section, we establish some inequalities associated with circular cone, which we believe will be useful for further analyzing the properties of $f^{\mathcal{L}_{\theta}}$ and proving the convergence of interior point methods for optimization problems involved in circular cones.

In [18], the author establishes the following results in the framework of second-order cone. More specifically, for $x \succeq_{\mathcal{L}_{45^{\circ}}} 0$ and $y \succeq_{\mathcal{L}_{45^{\circ}}} 0$, then

(a) $\operatorname{det}(e+x)^{1 / 2} \geq 1+\operatorname{det}(x)^{1 / 2}$,

(b) $\operatorname{det}(x+y) \geq \operatorname{det}(x)+\operatorname{det}(y)$,

(c) $\operatorname{det}(\alpha x+(1-\alpha) y) \geq \alpha^{2} \operatorname{det}(x)+(1-\alpha)^{2} \operatorname{det}(y), \forall \alpha \in[0,1]$,

(d) $\operatorname{det}(e+x+y) \leq \operatorname{det}(e+x) \operatorname{det}(e+y)$, 
(e) If $x \succeq_{\mathcal{L}_{45^{\circ}}} y \succeq_{\mathcal{L}_{45^{\circ}}} 0$, then $\operatorname{det}(x) \geq \operatorname{det}(y), \operatorname{tr}(x) \geq \operatorname{tr}(y)$, and $\lambda_{i}(x) \geq \lambda_{i}(y)$ for $i=1,2$,

(f) $\operatorname{tr}(x+y)=\operatorname{tr}(x)+\operatorname{tr}(y)$ and $\operatorname{det}(\gamma x)=\gamma^{2} \operatorname{det}(x)$ for all $\gamma \in \mathbb{R}$.

In the following, we show that, in the framework of circular cone, the above inequalities can be classified into three categories. The first class holds independent of the angle, e.g., (a); the second class holds dependent on the angle, e.g., (b)-(e); the third class fails no matter what value of the angle is chosen, e.g., (f).

Theorem 3.1 Let $x=\left(x_{1}, x_{2}\right) \in \mathbb{R} \times \mathbb{R}^{n-1}$ possess spectral factorization associated with circular cone given as in (1). Then

(a) $[\operatorname{det}(e+x)]^{1 / 2} \geq 1+\operatorname{det}(x)^{1 / 2}$ for all $x \in \mathcal{L}_{\theta}$;

(b) If $x \succeq \mathcal{L}_{\theta} y$, then $\lambda_{1}(x) \geq \lambda_{1}(y)$.

Proof (a) Note that $\operatorname{det}(x) \geq 0$ and $\operatorname{det}(e+x) \geq 0$ since $x, x+e \in \mathcal{L}_{\theta}$. Therefore,

$$
\begin{aligned}
& {[\operatorname{det}(e+x)]^{1 / 2} \geq 1+\operatorname{det}(x)^{1 / 2} } \\
& \Longleftrightarrow \quad \operatorname{det}(e+x) \geq 1+2 \operatorname{det}(x)^{1 / 2}+\operatorname{det}(x) \\
& \Longleftrightarrow \quad \lambda_{1}(e+x) \lambda_{2}(e+x) \geq 1+2 \sqrt{\lambda_{1}(x) \lambda_{2}(x)}+\lambda_{1}(x) \lambda_{2}(x) \\
& \Longleftrightarrow \quad\left(x_{1}+1-\left\|x_{2}\right\| \cot \theta\right)\left(x_{1}+1+\left\|x_{2}\right\| \tan \theta\right) \geq 1+2 \sqrt{\lambda_{1}(x) \lambda_{2}(x)}+\lambda_{1}(x) \lambda_{2}(x) \\
& \Longleftrightarrow \quad\left(\lambda_{1}(x)+1\right)\left(\lambda_{2}(x)+1\right) \geq 1+2 \sqrt{\lambda_{1}(x) \lambda_{2}(x)}+\lambda_{1}(x) \lambda_{2}(x) \\
& \Longleftrightarrow \quad \lambda_{1}(x) \lambda_{2}(x)+\lambda_{1}(x)+\lambda_{2}(x)+1 \geq 1+2 \sqrt{\lambda_{1}(x) \lambda_{2}(x)}+\lambda_{1}(x) \lambda_{2}(x) \\
& \Longleftrightarrow \quad \lambda_{1}(x)+\lambda_{2}(x) \geq 2 \sqrt{\lambda_{1}(x) \lambda_{2}(x)} \\
& \Longleftrightarrow \quad \frac{\lambda_{1}(x)+\lambda_{2}(x)}{2} \geq \sqrt{\lambda_{1}(x) \lambda_{2}(x)} .
\end{aligned}
$$

Hence, to prove the desired result, it suffices to show that

$$
\frac{\lambda_{1}(x)+\lambda_{2}(x)}{2} \geq \sqrt{\lambda_{1}(x) \lambda_{2}(x)}
$$

which is clearly true by the arithmetic mean-geometric mean (AM-GM) inequality.

(b) Since $x-y \in \mathcal{L}_{\theta}$, we know

$$
x_{1}-y_{1} \geq\left\|x_{2}-y_{2}\right\| \cot \theta \geq\left[\left\|x_{2}\right\|-\left\|y_{2}\right\|\right] \cot \theta,
$$

i.e., $\lambda_{1}(x)=x_{1}-\left\|x_{2}\right\| \cot \theta \geq y_{1}-\left\|y_{2}\right\| \cot \theta=\lambda_{1}(y)$.

Theorem 3.2 Let $x=\left(x_{1}, x_{2}\right) \in \mathbb{R} \times \mathbb{R}^{n-1}$ possess spectral factorization associated with circular cone given as in (1). Then the following hold.

(a) For all $x, y \in \mathcal{L}_{\theta}$,

$$
\operatorname{det}(x+y) \geq \operatorname{det}(x)+\operatorname{det}(y)+\left(\left\|x_{2}\right\|^{2}+\left\|y_{2}\right\|^{2}\right) \csc ^{2} \theta-\left(x_{1}^{2}+y_{1}^{2}\right) \sec ^{2} \theta .
$$

In particular, when $\theta \in\left(0,45^{\circ}\right]$, we have

$$
\operatorname{det}(x+y) \geq \operatorname{det}(x)+\operatorname{det}(y)
$$


(b) For all $x, y \in \mathcal{L}_{\theta}$ and $\alpha \in[0,1]$,

$$
\begin{aligned}
& \operatorname{det}(\alpha x+(1-\alpha) y) \\
& \geq \alpha^{2} \operatorname{det}(x)+(1-\alpha)^{2} \operatorname{det}(y)+\left(\alpha^{2}\left\|x_{2}\right\|^{2}+(1-\alpha)^{2}\left\|y_{2}\right\|^{2}\right) \csc ^{2} \theta \\
& \quad-\left(\alpha^{2} x_{1}^{2}+(1-\alpha)^{2} y_{1}^{2}\right) \sec ^{2} \theta .
\end{aligned}
$$

In particular, when $\theta \in\left(0,45^{\circ}\right]$, we have

$$
\operatorname{det}(\alpha x+(1-\alpha) y) \geq \alpha^{2} \operatorname{det}(x)+(1-\alpha)^{2} \operatorname{det}(y) .
$$

(c) If $x, y \in \mathcal{L}_{\theta}$ and $\theta \in\left[45^{\circ}, 90^{\circ}\right)$, then

$$
\operatorname{det}(e+x+y) \leq \operatorname{det}(e+x) \operatorname{det}(e+y) .
$$

(d) If $x \succeq_{\mathcal{L}_{\theta}} y \succeq_{\mathcal{L}_{\theta}} 0$ and $\theta \in\left(0,45^{\circ}\right]$, then

$$
\lambda_{2}(x) \geq \lambda_{2}(y), \quad \operatorname{det}(x) \geq \operatorname{det}(y), \quad \text { and } \quad \operatorname{tr}(x) \geq \operatorname{tr}(y) .
$$

Proof (a) Notice that

$$
\begin{aligned}
& \operatorname{det}(x+y) \\
& \quad=\lambda_{1}(x+y) \cdot \lambda_{2}(x+y) \\
& \quad=\left(x_{1}+y_{1}-\left\|x_{2}+y_{2}\right\| \cot \theta\right)\left(x_{1}+y_{1}+\left\|x_{2}+y_{2}\right\| \tan \theta\right) \\
& =\left(x_{1}+y_{1}\right)^{2}+\left(x_{1}+y_{1}\right)\left\|x_{2}+y_{2}\right\| \tan \theta-\left(x_{1}+y_{1}\right)\left\|x_{2}+y_{2}\right\| \cot \theta-\left\|x_{2}+y_{2}\right\|^{2}
\end{aligned}
$$

and

$$
\begin{aligned}
& \operatorname{det}(x)+\operatorname{det}(y) \\
& \quad=\lambda_{1}(x) \lambda_{2}(x)+\lambda_{1}(y) \lambda_{2}(y) \\
& =\left(x_{1}-\left\|x_{2}\right\| \cot \theta\right)\left(x_{1}+\left\|x_{2}\right\| \tan \theta\right)+\left(y_{1}-\left\|y_{2}\right\| \cot \theta\right)\left(y_{1}+\left\|y_{2}\right\| \tan \theta\right) \\
& =x_{1}^{2}+x_{1}\left\|x_{2}\right\| \tan \theta-x_{1}\left\|x_{2}\right\| \cot \theta-\left\|x_{2}\right\|^{2}+y_{1}^{2}+y_{1}\left\|y_{2}\right\| \tan \theta-y_{1}\left\|y_{2}\right\| \cot \theta-\left\|y_{2}\right\|^{2} \\
& =x_{1}^{2}+y_{1}^{2}+x_{1}\left\|x_{2}\right\| \tan \theta+y_{1}\left\|y_{2}\right\| \tan \theta-x_{1}\left\|x_{2}\right\| \cot \theta-y_{1}\left\|y_{2}\right\| \cot \theta-\left\|x_{2}\right\|^{2}-\left\|y_{2}\right\|^{2} .
\end{aligned}
$$

Then we have

$$
\begin{aligned}
& \operatorname{det}(x+y)-\operatorname{det}(x)-\operatorname{det}(y) \\
& =2 x_{1} y_{1}-2 x_{2}^{T} y_{2}+\left(x_{1}\left\|x_{2}+y_{2}\right\|+y_{1}\left\|x_{2}+y_{2}\right\|-x_{1}\left\|x_{2}\right\|-y_{1}\left\|y_{2}\right\|\right) \tan \theta \\
& \quad-\left(x_{1}\left\|x_{2}+y_{2}\right\|+y_{1}\left\|x_{2}+y_{2}\right\|-x_{1}\left\|x_{2}\right\|-y_{1}\left\|y_{2}\right\|\right) \cot \theta .
\end{aligned}
$$

Using $x, y \in \mathcal{L}_{\theta}$ (and hence $x+y \in \mathcal{L}_{\theta}$ ) gives

$$
\begin{aligned}
& x_{1} \tan \theta \geq\left\|x_{2}\right\|, \quad-x_{1} \tan \theta \leq-\left\|x_{2}\right\|, \quad x_{1} \geq\left\|x_{2}\right\| \cot \theta, \quad-x_{1} \leq-\left\|x_{2}\right\| \cot \theta, \\
& -\left(x_{1}+y_{1}\right) \leq-\left\|x_{2}+y_{2}\right\| \cot \theta .
\end{aligned}
$$


Thus,

$$
\begin{aligned}
& \operatorname{det}(x+y)-\operatorname{det}(x)-\operatorname{det}(y) \\
& \geq 2 x_{1} y_{1}-2 x_{2}^{T} y_{2}+\left\|x_{2}\right\|\left\|x_{2}+y_{2}\right\|+\left\|y_{2}\right\|\left\|x_{2}+y_{2}\right\|-x_{1}^{2} \tan ^{2} \theta-y_{1}^{2} \tan ^{2} \theta \\
& \quad-x_{1}\left(x_{1}+y_{1}\right)-y_{1}\left(x_{1}+y_{1}\right)+\left\|x_{2}\right\|^{2} \cot ^{2} \theta+\left\|y_{2}\right\|^{2} \cot ^{2} \theta \\
&= 2 x_{1} y_{1}-2 x_{2}^{T} y_{2}+\left\|x_{2}+y_{2}\right\|\left(\left\|x_{2}\right\|+\left\|y_{2}\right\|\right)-\left(x_{1}^{2}+y_{1}^{2}\right) \tan ^{2} \theta \\
& \quad-\left(x_{1}+y_{1}\right)^{2}+\left(\left\|x_{2}\right\|^{2}+\left\|y_{2}\right\|^{2}\right) \cot ^{2} \theta \\
& \geq\left\|x_{2}+y_{2}\right\|^{2}-\left(x_{1}^{2}+y_{1}^{2}\right) \tan ^{2} \theta-x_{1}^{2}-y_{1}^{2}-2 x_{2}^{T} y_{2}+\left(\left\|x_{2}\right\|^{2}+\left\|y_{2}\right\|^{2}\right) \cot ^{2} \theta \\
&=\left\|x_{2}\right\|^{2}+\left\|y_{2}\right\|^{2}-\left(x_{1}^{2}+y_{1}^{2}\right) \tan ^{2} \theta-x_{1}^{2}-y_{1}^{2}+\left(\left\|x_{2}\right\|^{2}+\left\|y_{2}\right\|^{2}\right) \cot ^{2} \theta \\
&=\left(\left\|x_{2}\right\|^{2}+\left\|y_{2}\right\|^{2}\right)\left(1+\cot ^{2} \theta\right)-\left(x_{1}^{2}+y_{1}^{2}\right)\left(1+\tan ^{2} \theta\right) \\
&=\left(\left\|x_{2}\right\|^{2}+\left\|y_{2}\right\|^{2}\right) \csc ^{2} \theta-\left(x_{1}^{2}+y_{1}^{2}\right) \sec ^{2} \theta,
\end{aligned}
$$

which is the desired result.

When $\theta \in\left(0,45^{\circ}\right]$, we know $\tan \theta \leq \cot \theta$. Since $x, y \in \mathcal{L}_{\theta}$, i.e., $x_{1} \geq\left\|x_{2}\right\| \cot \theta$ and $y_{1} \geq\left\|y_{2}\right\| \cot \theta$, there exist $a, b \geq 0$ such that $x_{1}=\left\|x_{2}\right\| \cot \theta+a$ and $y_{1}=\left\|y_{2}\right\| \cot \theta+b$. Hence,

$$
\begin{aligned}
\operatorname{det}(x+y)-\operatorname{det}(x)-\operatorname{det}(y) & \\
= & 2 x_{1} y_{1}-2 x_{2}^{T} y_{2}+\left(x_{1}\left\|x_{2}+y_{2}\right\|+y_{1}\left\|x_{2}+y_{2}\right\|-x_{1}\left\|x_{2}\right\|-y_{1}\left\|y_{2}\right\|\right) \tan \theta \\
& \quad-\left(x_{1}\left\|x_{2}+y_{2}\right\|+y_{1}\left\|x_{2}+y_{2}\right\|-x_{1}\left\|x_{2}\right\|-y_{1}\left\|y_{2}\right\|\right) \cot \theta \\
= & \left(\left\|x_{2}\right\|+\left\|y_{2}\right\|\right)\left[\left\|x_{2}\right\|+\left\|y_{2}\right\|-\left\|x_{2}+y_{2}\right\|\right] \cot ^{2} \theta \\
& \quad+\left\|x_{2}+y_{2}\right\|\left(\left\|x_{2}\right\|+\left\|y_{2}\right\|-\left\|x_{2}+y_{2}\right\|\right)+2 a b \\
& +a \cot \theta\left(\left\|y_{2}\right\|+\left\|x_{2}\right\|-\left\|x_{2}+y_{2}\right\|\right)+a \tan \theta\left(\left\|y_{2}\right\| \cot ^{2} \theta+\left\|x_{2}+y_{2}\right\|-\left\|x_{2}\right\|\right) \\
& +b \cot \theta\left(\left\|y_{2}\right\|+\left\|x_{2}\right\|-\left\|x_{2}+y_{2}\right\|\right)+b \tan \theta\left(\left\|x_{2}\right\| \cot ^{2} \theta+\left\|x_{2}+y_{2}\right\|-\left\|y_{2}\right\|\right) \\
\geq & 0,
\end{aligned}
$$

where the last step is due to $\left\|x_{2}\right\|+\left\|y_{2}\right\| \geq\left\|x_{2}+y_{2}\right\|,\left\|x_{2}\right\| \cot ^{2} \theta+\left\|x_{2}+y_{2}\right\|-\left\|y_{2}\right\| \geq$ $\left\|x_{2}\right\|+\left\|x_{2}+y_{2}\right\|-\left\|y_{2}\right\| \geq 0$, and $\left\|y_{2}\right\| \cot ^{2} \theta+\left\|x_{2}+y_{2}\right\|-\left\|x_{2}\right\| \geq\left\|y_{2}\right\|+\left\|x_{2}+y_{2}\right\|-\left\|x_{2}\right\| \geq 0$ since $\cot \theta \geq 1$, due to $\theta \in\left(0,45^{\circ}\right]$.

(b) The result follows from the fact that $\operatorname{det}(\gamma x)=\gamma^{2} \operatorname{det}(x)$ for all $\gamma \geq 0$.

(c) Since $\theta \in\left[45^{\circ}, 90^{\circ}\right), \cot \theta \leq 1$. For $x, y \in \mathcal{L}_{\theta}$, there exist two nonnegative scalars $a, b \geq 0$ such that $x_{1}=\left\|x_{2}\right\| \cot \theta+a$ and $y_{1}=\left\|y_{2}\right\| \cot \theta+b$. This implies

$$
\begin{aligned}
\operatorname{det}(e+x) & =\left(x_{1}+1-\left\|x_{2}\right\| \cot \theta\right)\left(x_{1}+1+\left\|x_{2}\right\| \tan \theta\right) \\
& =(a+1)(\cot \theta+\tan \theta)\left\|x_{2}\right\|+(a+1)^{2}, \\
\operatorname{det}(e+y) & =\left(y_{1}+1-\left\|y_{2}\right\| \cot \theta\right)\left(y_{1}+1+\left\|y_{2}\right\| \tan \theta\right) \\
& =(b+1)(\cot \theta+\tan \theta)\left\|y_{2}\right\|+(b+1)^{2} .
\end{aligned}
$$


Thus, we obtain

$$
\begin{aligned}
& \operatorname{det}(e+x) \operatorname{det}(e+y) \\
& =(a+1)(b+1)(\cot \theta+\tan \theta)^{2}\left\|x_{2}\right\|\left\|y_{2}\right\|+(a+1)(b+1)^{2}(\cot \theta+\tan \theta)\left\|x_{2}\right\| \\
& \quad+(a+1)^{2}(b+1)(\cot \theta+\tan \theta)\left\|y_{2}\right\|+(a+1)^{2}(b+1)^{2} .
\end{aligned}
$$

On the other hand,

$$
\begin{aligned}
& \operatorname{det}(e+x+y) \\
& =\left(x_{1}+y_{1}+1-\left\|x_{2}+y_{2}\right\| \cot \theta\right)\left(x_{1}+y_{1}+1+\left\|x_{2}+y_{2}\right\| \tan \theta\right) \\
& =\left(\left[\left\|x_{2}\right\|+\left\|y_{2}\right\|-\left\|x_{2}+y_{2}\right\|\right] \cot \theta+(a+b+1)\right) \\
& \times\left(\left[\left\|x_{2}\right\|+\left\|y_{2}\right\|\right] \cot \theta+\left\|x_{2}+y_{2}\right\| \tan \theta+(a+b+1)\right) \\
& =\left(\left\|x_{2}\right\|+\left\|y_{2}\right\|-\left\|x_{2}+y_{2}\right\|\right)\left(\left\|x_{2}\right\|+\left\|y_{2}\right\|\right) \cot ^{2} \theta \\
& +\left(\left\|x_{2}\right\|+\left\|y_{2}\right\|-\left\|x_{2}+y_{2}\right\|\right)\left\|x_{2}+y_{2}\right\|+(a+b+1)\left(\left\|x_{2}\right\|+\left\|y_{2}\right\|-\left\|x_{2}+y_{2}\right\|\right) \cot \theta \\
& +(a+b+1)\left(\left\|x_{2}\right\|+\left\|y_{2}\right\|\right) \cot \theta+(a+b+1)\left\|x_{2}+y_{2}\right\| \tan \theta+(a+b+1)^{2} \\
& =2 \cot ^{2} \theta\left\|x_{2}\right\|\left\|y_{2}\right\|+2(a+b+1) \cot \theta\left\|x_{2}\right\|+2(a+b+1) \cot \theta\left\|y_{2}\right\|+(a+b+1)^{2} \\
& +\left[\left\|x_{2}\right\|^{2}+\left\|y_{2}\right\|^{2}\right] \cot ^{2} \theta+\left(1-\cot ^{2} \theta\right)\left\|x_{2}+y_{2}\right\|\left(\left\|x_{2}\right\|+\left\|y_{2}\right\|\right) \\
& +(a+b+1)(\tan \theta-\cot \theta)\left\|x_{2}+y_{2}\right\|-\left\|x_{2}+y_{2}\right\|^{2} \\
& \leq 2 \cot ^{2} \theta\left\|x_{2}\right\|\left\|y_{2}\right\|+2(a+b+1) \cot \theta\left\|x_{2}\right\|+2(a+b+1) \cot \theta\left\|y_{2}\right\|+(a+b+1)^{2} \\
& +\left[\left\|x_{2}\right\|^{2}+\left\|y_{2}\right\|^{2}\right] \cot ^{2} \theta+\left(1-\cot ^{2} \theta\right)\left(\left\|x_{2}\right\|+\left\|y_{2}\right\|\right)^{2} \\
& +(a+b+1)(\tan \theta-\cot \theta)\left(\left\|x_{2}\right\|+\left\|y_{2}\right\|\right)-\left(\left\|x_{2}\right\|^{2}+\left\|y_{2}\right\|^{2}\right)+2\left\|x_{2}\right\|\left\|y_{2}\right\| \\
& =2 \cot ^{2} \theta\left\|x_{2}\right\|\left\|y_{2}\right\|+(a+b+1)(\cot \theta+\tan \theta)\left\|x_{2}\right\|+(a+b+1)(\cot \theta+\tan \theta)\left\|y_{2}\right\| \\
& +(a+b+1)^{2}+\left(1-\cot ^{2} \theta\right)\left[\left(\left\|x_{2}\right\|+\left\|y_{2}\right\|\right)^{2}-\left(\left\|x_{2}\right\|^{2}+\left\|y_{2}\right\|^{2}\right)\right]+2\left\|x_{2}\right\|\left\|y_{2}\right\| \\
& =4\left\|x_{2}\right\|\left\|y_{2}\right\|+(a+b+1)(\cot \theta+\tan \theta)\left\|x_{2}\right\|+(a+b+1)(\operatorname{ctan} \theta+\tan \theta)\left\|y_{2}\right\| \\
& +(a+b+1)^{2} \text {. }
\end{aligned}
$$

Note that $(a+1)(b+1)(\cot \theta+\tan \theta)^{2} \geq(\cot \theta+\tan \theta)^{2} \geq 4$ and

$$
\begin{aligned}
& (a+1)(b+1)^{2} \geq a+b+1, \\
& (a+1)^{2}(b+1) \geq a+b+1, \\
& (a+1)^{2}(b+1)^{2} \geq(a+b+1)^{2} .
\end{aligned}
$$

Hence, comparing (22) and (23) yields

$$
\operatorname{det}(e+x+y) \leq \operatorname{det}(e+x) \operatorname{det}(e+y)
$$

(d) For $\theta \in\left(0,45^{\circ}\right]$, since $\cot \theta \geq \tan \theta$ and $x-y \in \mathcal{L}_{\theta}$, we know

$$
x_{1}-y_{1} \geq\left\|x_{2}-y_{2}\right\| \cot \theta \geq\left\|x_{2}-y_{2}\right\| \tan \theta \geq\left[\left\|y_{2}\right\|-\left\|x_{2}\right\|\right] \tan \theta,
$$


which means

$$
\lambda_{2}(x)=x_{1}+\left\|x_{2}\right\| \tan \theta \geq y_{1}+\left\|y_{2}\right\| \tan \theta=\lambda_{2}(y) .
$$

This together with the fact $\lambda_{1}(x) \geq \lambda_{1}(y)$ by Part (b) in Theorem 3.1 and $\lambda_{i}(x), \lambda_{i}(y) \geq 0$ for $i=1,2$ (due to $x, y \in \mathcal{L}_{\theta}$ ) further yields

$$
\operatorname{det}(x)=\lambda_{1}(x) \lambda_{2}(x) \geq \lambda_{1}(y) \lambda_{2}(y)=\operatorname{det}(y) .
$$

Meanwhile, we obtain

$$
\operatorname{tr}(x)=\lambda_{1}(x)+\lambda_{2}(x) \geq \lambda_{1}(y)+\lambda_{2}(y)=\operatorname{tr}(y) .
$$

Here are some remarks for Theorem 3.2.

(i) Inequality (19) fails when $\theta \in\left(45^{\circ}, 90^{\circ}\right)$. For example, let $x=(1,3,4), y=(1,-3,-4)$, and $\cot \theta=0.1$. Then $\operatorname{det}(x)=\operatorname{det}(y)=51 / 2$ and $\operatorname{det}(x+y)=4$, which says $\operatorname{det}(x+y)=4<51=\operatorname{det}(x)+\operatorname{det}(y)$.

(ii) Inequality (20) fails when $\theta \in\left(0,45^{\circ}\right)$. For example, let $x=(3 / 10,1 / 10)$, $y=(3 / 10,-1 / 10)$, and $\cot \theta=2$. Then $\operatorname{det}(e+x+y)=(1.6)^{2}>(1.485)^{2}=\operatorname{det}(e+x) \operatorname{det}(e+y)$.

(iii) Inequality (21) fails when $\theta \in\left(45^{\circ}, 90^{\circ}\right)$. For example, for $x=(1.1,1), y=(1,2)$, and $\cot \theta=0.1$. Then $x \succeq_{\mathcal{L}_{\theta}} y, \lambda_{2}(x)=11.1<21=\lambda_{2}(y)$, $\operatorname{det}(x)=(1.1-0.1)(1.1+10)=11.1<16.8=(1-0.2)(1+20)=\operatorname{det}(y)$, and $\operatorname{tr}(x)=12.1<21.8=\operatorname{tr}(y)$.

Next, let us move from inequalities to equalities. In particular, we focus on two identities in the framework of second-order cone as below

$$
\operatorname{tr}(x+y)=\operatorname{tr}(x)+\operatorname{tr}(y) \quad \text { and } \quad \operatorname{det}(\gamma x)=\gamma^{2} \operatorname{det}(x), \quad \forall \gamma \in \mathbb{R} .
$$

But these two identities fail to hold in the circular cone setting no matter what value of the angle is chosen. In fact, in the second-order cone case,

$$
\operatorname{tr}(x)=2 x_{1} \quad \text { and } \quad \operatorname{det}(x)=x_{1}^{2}-\left\|x_{2}\right\|^{2} .
$$

Hence, (24) holds trivially. For the circular cone setting, we have

$$
\operatorname{tr}(x)=2 x_{1}+\left\|x_{2}\right\|(\tan \theta-\cot \theta) \quad \text { and } \quad \operatorname{det}(x)=\left(x_{1}-\left\|x_{2}\right\| \cot \theta\right)\left(x_{1}+\left\|x_{2}\right\| \tan \theta\right)
$$

Thus, $\operatorname{tr}(x)$ is not linear any more, i.e., $\operatorname{tr}(x+y) \neq \operatorname{tr}(x)+\operatorname{tr}(y)$; e.g., for $x=(1,2)$ and $y=(1,-2)$, and $\cot \theta=1 / 2($ or $\cot \theta=2)$. Then

$$
\operatorname{tr}(x+y)=4 \neq 10=\operatorname{tr}(x)+\operatorname{tr}(y) \quad(\text { or } \operatorname{tr}(x+y)=4 \neq-2=\operatorname{tr}(x)+\operatorname{tr}(y)) .
$$

In addition, $\operatorname{det}(\gamma x)=\gamma^{2} \operatorname{det}(x)$ holds as $\gamma \geq 0$ but not true as $\gamma<0$; e.g., for $x=(3,4)$, $\gamma=-2$, and $\cot \theta=1 / 2($ or $\cot \theta=2)$, then

$$
\operatorname{det}(-2 x)=-100 \neq 44=(-2)^{2} \operatorname{det}(x) \quad\left(\text { or } \operatorname{det}(-2 x)=44 \neq-100=(-2)^{2} \operatorname{det}(x)\right) .
$$


The precise relationship between $\operatorname{tr}(x+y)$ and $\operatorname{tr}(x)+\operatorname{tr}(y)$ is provided as below.

\section{Theorem 3.3}

$$
\operatorname{tr}(x+y) \begin{cases}\geq \operatorname{tr}(x)+\operatorname{tr}(y) & \text { as } \theta \in\left(0,45^{\circ}\right] \\ \leq \operatorname{tr}(x)+\operatorname{tr}(y) & \text { as } \theta \in\left[45^{\circ}, 90^{\circ}\right)\end{cases}
$$

Proof The result follows from the fact that

$$
\operatorname{tr}(x+y)-\operatorname{tr}(x)-\operatorname{tr}(y)=\left[\left\|x_{2}\right\|+\left\|y_{2}\right\|-\left\|x_{2}+y_{2}\right\|\right](\cot \theta-\tan \theta) .
$$

Note that $\operatorname{tr}(x)$ is positively homogeneous, i.e., $\operatorname{tr}(\gamma x)=\gamma \operatorname{tr}(x)$ for all $\gamma \geq 0$. This together with Theorem 3.3 yields the following result.

Corollary 3.1 The trace $\operatorname{tr}(x)$ is concave as $\theta \in\left(0,45^{\circ}\right]$ and is convex as $\theta \in\left[45^{\circ}, 90^{\circ}\right)$.

These results further indicate that the angle plays an essential role for a circular cone. As in symmetric cone optimization, we believe that these inequalities about $\operatorname{det}(x)$ and $\operatorname{tr}(x)$ are key ingredients in penalty and barrier functions which can be adapted in designing barrier and penalty algorithms (including interior point algorithm) for circular cone optimization. This merits our further research.

Competing interests

The authors declare that they have no competing interests.

Authors' contributions

All authors read and approved the final manuscript.

\section{Author details}

'Department of Mathematics, School of Science, Shandong University of Technology, Zibo, 255049, P.R. China.

${ }^{2}$ Department of Mathematics, National Taiwan Normal University, Taipei, 11677, Taiwan.

\section{Authors' information}

The second author is a member of Mathematics Division, National Center for Theoretical Sciences, Taipei Office.

\section{Acknowledgements}

We are gratefully indebted to anonymous referees for their valuable suggestions that helped us to essentially improve the original presentation of the paper. The first author's work is supported by the National Natural Science Foundation of China $(11101248,11271233)$ and Shandong Province Natural Science Foundation (ZR2010AQ026, ZR2012AM016). The second author's work is supported by the National Science Council of Taiwan.

Received: 17 July 2013 Accepted: 13 November 2013 Published: 04 Dec 2013

\section{References}

1. Matsukawa, Y, Yoshise, A: A primal barrier function Phase I algorithm for nonsymmetric conic optimization problems. Jpn. J. Ind. Appl. Math. 29, 499-517, (2012)

2. Nesterov, $Y$ : Towards nonsymmetric conic optimization. CORE (2006)

3. Skajaa, A, Jørgensen, JB, Hansen, PC: On implementing a homogeneous interior-point algorithm for nonsymmetric conic optimization. DTU Informatics, Building 321 (2011)

4. Skajaa, A, Ye, Y-Y: A homogeneous interior-point algorithm for nonsymmetric convex conic optimization (2012, submitted manuscript)

5. Faraut, U, Korányi, A: Analysis on Symmetric Cones. Oxford Mathematical Monographs. Oxford University Press, New York (1994)

6. Dattorro, J: Convex Optimization and Euclidean Distance Geometry. Meboo Publishing, Palo Alto (2005)

7. Zhou, J-C, Chen, J-S: Properties of circular cone and spectral factorization associated with circular cone. J. Nonlinear Convex Anal. 14, 807-816 (2013)

8. Zhou, J-C, Chen, J-S: On the vector-valued functions associated with circular cones (2013, submitted manuscript)

9. Zhou, J-C, Chen, J-S: Circular cone monotonicity and second order differentiability of vector-valued functions associated with circular cones (2013, submitted manuscript) 
10. Chang, Y-L, Yang, C-Y, Chen, J-S: Smooth and nonsmooth analysis of vector-valued functions associated with circular cones. Nonlinear Anal., Theory Methods Appl. 85, 160-173 (2013)

11. Ko, C-H, Chen, J-S: Optimal grasping manipulation for multifingered robots using semismooth Newton method. Math. Probl. Eng. 2013, Article ID 681710 (2013)

12. Pan, S-H, Chiang, Y, Chen, J-S: SOC-monotone and SOC-convex functions v.s. matrix-monotone and matrix-convex functions. Linear Algebra Appl. 437(5), 1264-1284 (2012)

13. Chen, J-S, Chen, X, Tseng, P: Analysis of nonsmooth vector-valued functions associated with second-order cone. Math. Program. 101(1), 95-117 (2004)

14. Chen, J-S, Pan, S-H: A survey on SOC complementarity functions and solution methods for SOCPs and SOCCPs. Pac. J. Optim. 8(1), 33-74 (2012)

15. Auslender, A: Penalty and barrier methods: a unified framework. SIAM J. Optim. 10, $211-230$ (1999)

16. Auslender, A, Ramirez, H: Penalty and barrier methods for convex semidefinite programming. Math. Methods Oper. Res. 63, 195-219 (2006)

17. Chang, Y-L, Chen, J-S: Convexity of symmetric cone trace functions in Euclidean Jordan algebras. J. Nonlinear Convex Anal. 14(1), 53-61 (2013)

18. Chen, J-S: The convex and monotone functions associated with second-order cone. Optimization 55(4), 363-385 (2006)

19. Chen, J-S, Chen, X, Pan, S-H, Zhang, J: Some characterizations for SOC-monotone and SOC-convex functions. J. Glob. Optim. 45(2), 259-279 (2009)

20. Chen, J-S, Liao, T-K, Pan, S-H: Using Schur complement theorem to prove convexity of some SOC-functions. J. Nonlinear Convex Anal. 13, 421-431 (2012)

10.1186/1029-242X-2013-571

Cite this article as: Zhou et al.: Circular cone convexity and some inequalities associated with circular cones. Journal of Inequalities and Applications 2013, 2013:571

\section{Submit your manuscript to a SpringerOpen ${ }^{\mathcal{O}}$ journal and benefit from:}

- Convenient online submission

- Rigorous peer review

- Immediate publication on acceptance

- Open access: articles freely available online

- High visibility within the field

- Retaining the copyright to your article 